\title{
Plasmonic rod dimers as elementary planar chiral meta-atoms
}

\author{
Sergei V. Zhukovsky, ${ }^{1,2}$ Christian Kremers, ${ }^{1}$ and Dmitry N. Chigrin ${ }^{1}$ \\ ${ }^{1)}$ Institute of High-Frequency and Communication Technology, Faculty of Electrical, Information and Media Engineering, \\ University of Wuppertal, Rainer-Gruenter-Str. 21, D-42119 Wuppertal, Germany \\ ${ }^{2)}$ Department of Physics and Institute for Optical Sciences, University of Toronto, 60 St. George Street, Toronto, Ontario M5S 1A7, \\ Canada
}

Electromagnetic response of metallic rod dimers is theoretically calculated for arbitrary planar arrangement of rods in the dimer. It is shown that dimers without an in-plane symmetry axis exhibit elliptical dichroism and act as "atoms" in planar chiral metamaterials. Due to a very simple geometry of the rod dimer, such planar metamaterials are much easier in fabrication than conventional split-ring or gammadion-type structures, and lend themselves to a simple analytical treatment based on coupled dipole model. Dependencies of metamaterial's directional asymmetry on the dimer's geometry are established analytically and confirmed in numerical simulations.

PACS numbers: 81.05.Xj, 78.67.Qa, 73.20.Mf

Metamaterials have attracted intense scientific interest in recent years for their unusual physical properties rare or absent in nature. One example of such properties is giant optical activity $\underline{1}$ in composite materials containing spiral-like or otherwise twisted elements ("meta-atoms"). Recently, planar chiral metamaterials (PCMs) were also introduced ${ }^{2}$. In PCMs, the meta-atoms possess two-dimensional (2D) rather than three-dimensional (3D) enantiomeric asymmetry (Fig. 1 $1 \mathrm{a}-\mathrm{b}$ ). PCMs are distinct from both 3D chiral and Faraday media in that their polarization eigenstates are co-rotating elliptical rather than counter-rotating elliptical or circular ${ }^{2}$. This leads to exotic polarization properties, e.g., asymmetry in transmission for left-handed (LH) vs. right-handed (RH) circularly polarized incident wave without nonreciprocity present in Faraday media. Such exotic properties, combined with the small dimensions of planar structures, make PCMs promising for polarization sensitive integrated optics applications.

Several different PCM designs have been proposed so far, the most notable example being the asymmetric chiral split rings ${ }^{3}$. Still, this geometry is complicated enough to make microscopic theoretical analysis difficult, and it can be shown that simpler geometries may be sufficient to achieve the desired chiral properties. Indeed, simulations reveal (Fig. 1F) that straightening the split-ring segments into two rods (preserving the segment's length and mutual orientation) results in similar transmission asymmetry for LH vs. RH circularly polarized light. Rods, unlike more complicated particles, lend themselves to a quite straightforward theoretical analysis (see, e.g. $\left.{ }^{4}\right)$. Hence it is of interest to explore the potential of tworod dimers as meta-atoms for PCMs. In addition, rod-like meta-atoms are much easier for fabrication than split-ring or gammadion structures, especially in the optical domain.

In this Letter, we show that double-rod plasmonic dimers can function as planar chiral meta-atoms. We find that the effective permittivity of a planar rod dimer supports elliptical dicroism necessary for PCM effects ${ }^{6}$. We confirm both analytically and numerically that any dimer with distinct $2 \mathrm{D}$ enantiomers intrinsically exhibits planar chiral behavior, and systematically investigate the relations between the strength of chiral properties and the dimer's geometrical parameters. By doing so, we propose a simple PCM design that lends itself to easy fabrication and simple analytical treatment. (a)
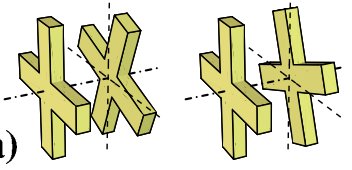

(b)

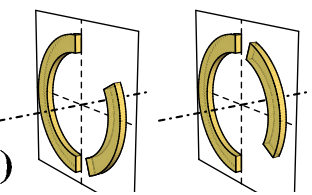

(c)

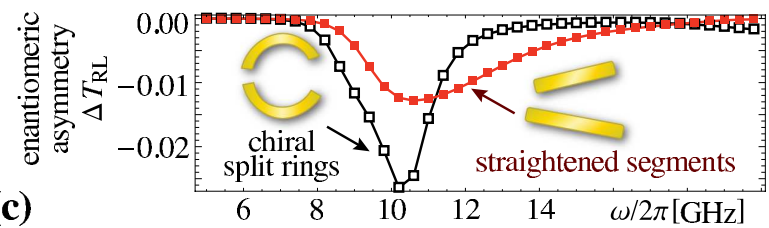

Figure 1. (Color online) Example of (a) 3D and (b) 2D enantiomeric meta-atoms; (c) difference in transmission for LH/RH-polarized wave for split-ring vs. "straighened split-ring" (rod dimer) structure.

We consider the structure shown in Fig.22 . Since both rods in the dimer are elongated, it is reasonable to start with the assumption that they respond to the external electric field $\mathbf{E}_{0}$ with an induced dipole moment $\mathbf{d}_{1,2}=\widehat{\alpha}_{1,2} \mathbf{E}_{0}$ where $\widehat{\alpha}_{j}$ is the polarizability tensor of a single rod. For a parallelepipedshaped rod one can follow the depolarization field approach ${ }^{7}$ to obtain polarizability in the form

$$
\widehat{\alpha}_{j}=\frac{f_{j} \vec{\mu}_{j} \otimes \vec{\mu}_{j}}{\omega_{j}^{2}-\omega^{2}-i \omega\left(\gamma_{j}+\delta_{j} \omega^{2}\right)} \equiv \alpha_{j} \vec{\mu}_{j} \otimes \vec{\mu}_{j}
$$

where the unit vector $\vec{\mu}_{j}$ denotes the rod orientation; $f_{j}, \omega_{j}$, $\gamma_{j}$, and $\delta_{j}$ are geometry-dependent parameters, and $\otimes$ stands for tensor (dyadic) product. Fig. 2 $2 \mathrm{~b}$ shows that Eq. (1) with parameters directly obtained from the rod dimensions and material properties provides a decent reproduction for the scattering cross-section spectrum of the rod. By letting the parameters $f_{j}, \omega_{j}, \gamma_{j}$, and $\delta_{j}$ be adjusted, a nearly perfect best-fit between the expression (1) and numerical simulations can be achieved. Also note that when a rod is bent to represent a ring segment, its scattering cross-section near its fundamental resonance does not undergo significant changes.

When there are two rods in a dimer, the external field $\mathbf{E}_{0}$ should be modified due to the presence of the neighboring rod. This results in a system of coupled equations for the dipole 


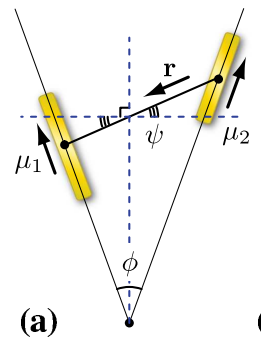

(b)

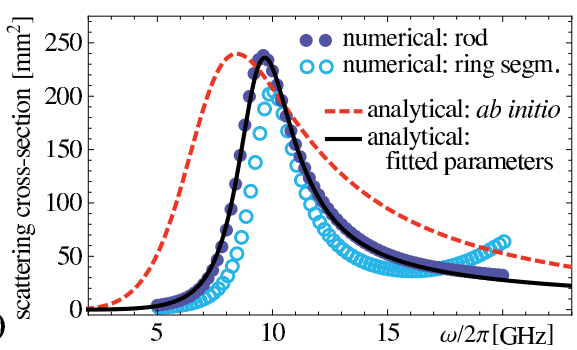

Figure 2. (Color online) (a) Geometry of an arbitrary planar rod dimer considered in the present study. (b) Scattering cross-section of a parallelepiped-shaped rod with dimensions $13 \times 0.8 \times 0.8 \mathrm{~mm}$ determined by numerical simulations and using Eq. (1), ab initio and using best-fit parameters. Numerical results for a $13 \mathrm{~mm}$ long ring segment are included for comparison.

moments of rods

$$
\mathbf{d}_{1,2}=\widehat{\alpha}_{1,2}\left[\mathbf{E}_{0}+\frac{k^{2}}{\varepsilon_{0}} \widehat{\mathbf{G}}(\mathbf{R}) \mathbf{d}_{2,1}\right]
$$

where $\mathbf{R}=R \mathbf{r}$ is the vector connecting the rods (Fig. 2a) and $\widehat{\mathbf{G}}$ is the dyadic Green's function in free space:

$$
\begin{gathered}
\widehat{\mathbf{G}}(\mathbf{R})=\frac{e^{i k R}}{4 \pi R}\left[\left(1+\frac{i k R-1}{k^{2} R^{2}}\right) \widehat{\mathrm{I}}+\frac{3-3 i k R-k^{2} R^{2}}{k^{2} R^{2}} \frac{\mathbf{R} \otimes \mathbf{R}}{R^{2}}\right] \\
\equiv G_{I} \widehat{\mathrm{I}}+G_{R} \mathbf{r} \otimes \mathbf{r} .
\end{gathered}
$$

with $\widehat{I}$ being the identity tensor. Solving Eqs. (2) with (3) for $\mathbf{d}_{1,2}$, one arrives at the total dipole moment $\mathbf{d}=\mathbf{d}_{1}+\mathbf{d}_{2}=$ $\widehat{\alpha}_{\text {eff }} \mathbf{E}_{0}$ of the dimer with effective polarizability tensor

$$
\begin{gathered}
\widehat{\alpha}_{\text {eff }}=\frac{\alpha_{1} \vec{\mu}_{1} \otimes \vec{\mu}_{1}+\alpha_{2} \vec{\mu}_{2} \otimes \vec{\mu}_{2}+\alpha_{1} \alpha_{2} \kappa\left(\vec{\mu}_{1} \otimes \vec{\mu}_{2}+\vec{\mu}_{2} \otimes \vec{\mu}_{1}\right)}{1-\alpha_{1} \alpha_{2} \kappa^{2}} \\
\equiv \alpha_{1}^{\text {eff }} \vec{\mu}_{1} \otimes \vec{\mu}_{1}+\alpha_{2}^{\text {eff }} \vec{\mu}_{2} \otimes \vec{\mu}_{2}+\alpha_{3}^{\text {eff }}\left(\vec{\mu}_{1} \otimes \vec{\mu}_{2}+\vec{\mu}_{2} \otimes \vec{\mu}_{1}\right)
\end{gathered}
$$

where $\kappa$ defines a coupling coefficient between the rods:

$$
\kappa=\frac{k^{2}}{\varepsilon_{0}}\left[G_{I}\left(\vec{\mu}_{1} \cdot \vec{\mu}_{2}\right)+G_{R}\left(\vec{\mu}_{1} \cdot \mathbf{r}\right)\left(\vec{\mu}_{2} \cdot \mathbf{r}\right)\right] .
$$

The effective permittivity tensor $\widehat{\varepsilon}_{\text {eff }}$ is derived from $\widehat{\alpha}_{\text {eff }}$ in Eq. (4) as $\widehat{\varepsilon}_{\text {eff }}=\widehat{\mathrm{I}}-\left(\varepsilon_{0} V_{\text {cell }}\right)^{-1} \widehat{\alpha}_{\text {eff }}$. In axial representation, $\widehat{\varepsilon}_{\text {eff }}$ can be expressed as

$$
\widehat{\varepsilon}_{\mathrm{eff}}=\widehat{\mathrm{I}}+\frac{1}{\varepsilon_{0} V_{\text {cell }}} \frac{\alpha_{1}^{\text {eff }}}{2}\left(\mathbf{c}_{+} \otimes \mathbf{c}_{-}+\mathbf{c}_{-} \otimes \mathbf{c}_{+}\right)
$$

with the complex vectors $\mathbf{c}_{ \pm}=\left(\vec{\mu}_{1}+\eta_{ \pm} \vec{\mu}_{2}\right)$ determining optical axes and $\eta_{ \pm}$given by

$$
\eta_{ \pm}=\frac{\alpha_{3}^{\text {eff }} \pm \sqrt{\left(\alpha_{3}^{\text {eff }}\right)^{2}-\alpha_{1}^{\text {eff }} \alpha_{2}^{\text {eff }}}}{\alpha_{1}^{\text {eff }}}
$$

The dielectric permittivity tensor (6) corresponds to an absorbing nonmagnetic crystal, which in general has two distinct eigenmodes with different polarizations, phase velocities and
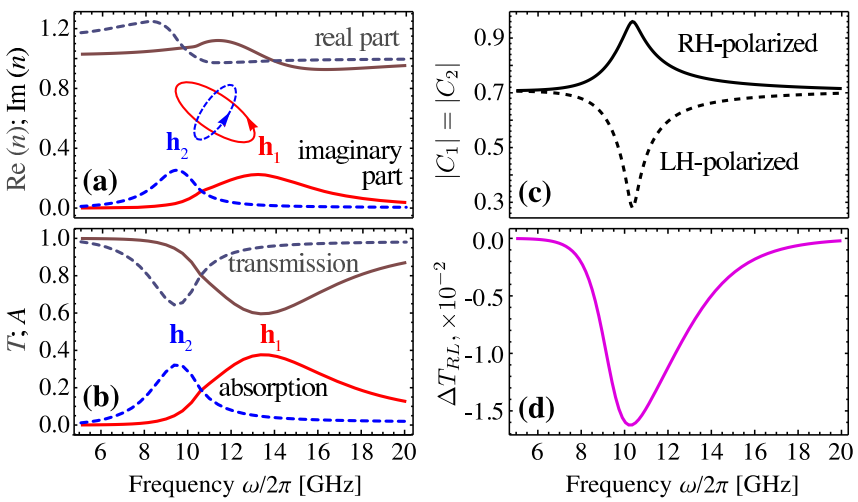

Figure 3. (Color online) (a) Refractive indices for two co-rotating elliptically polarized eigenwaves [eigenvectors $\mathbf{h}_{1,2}$ of the tensor $\widehat{N}_{H}$ in Eq. [8] in the effective medium given by Eq. [6]. (b) Transmission and absorption for incident wave with polarization coincident with $\mathbf{h}_{1,2}$. (c) Absolute value of the complex projection coefficients $C_{1,2}$ [see Eq. 9] for RH (solid) and LH (dashed) circularly polarized incident wave. (d) Analytically calculated difference in transmission for LH/RH-polarized incident wave. Here $a_{1}=13 \mathrm{~mm}, a_{2}=10 \mathrm{~mm}$, $d=10 \mathrm{~mm}, \phi=45^{\circ}$, and $\psi=0^{\circ}$

absorption coefficients $\stackrel{8}{-}$. The medium's eigenmodes $\mathbf{h}_{1,2}$ and their associated refractive indices $n_{1,2}$ are given by eigenvectors and eigenvalues of the refractive index tensor ${ }^{9}$

$$
\widehat{N}_{H}=\left[\left(-\mathbf{n}^{\times} \widehat{\varepsilon}_{\mathrm{eff}}^{-1} \mathbf{n}^{\times}\right)^{-}\right]^{-1 / 2} .
$$

Here $\mathbf{n}$ is an unit vector of the wave normal, the operator $\mathbf{n}^{\times}$is defined as $\left(\mathbf{n}^{\times}\right) \mathbf{u}=[\mathbf{n} \times \mathbf{u}]$, and $\widehat{A}^{-}$denotes a pseudoinverse tensor to $\widehat{A}$.

In Fig. 3 a the real and imaginary parts of $n_{1,2}$ are shown for propagation direction orthogonal to the meta-atoms plane. One can clearly see that absorption bands for the two eigenmodes are different, which is characteristic for dichroic media. Fig. 3 b shows the transmission and absorption spectra of the medium given by Eq. (6) for an incident wave with polarization coincident with the eigenmodes $\left(\mathbf{h}_{1,2}\right)$. We calculate the transmission spectrum from $\widehat{\varepsilon}_{\text {eff }}$ using generalized transfer matrix techniques (see, e.g.,$\frac{10}{}$ ). Due to the small variation in the real part of the refractive indices, reflection from such an effective medium slab is fairly small, and the dips seen in the transmission spectra are primarily due to absorption.

For an arbitrarily polarized incident wave, the slab acts as an absorbing polarization filter splitting the incident field $\mathbf{H}$ into two waves with polarizations parallel to the crystal eigenvectors $\mathbf{h}_{1,2}$, namely

$$
\mathbf{H}=C_{1} \mathbf{h}_{1}+C_{2} \mathbf{h}_{2},
$$

where $C_{1,2}$ are complex projection coefficients. The transmission of the effective medium is then determined by the relations (i) between complex projection coefficients $C_{1,2}$ and (ii) between absorption coefficients for the eigenwaves.

Fig $3 \mathrm{r}$ shows the projection coefficients $C_{1,2}$ for circularly polarized incident waves. In this case, it can be seen that 
(a) distance between rods

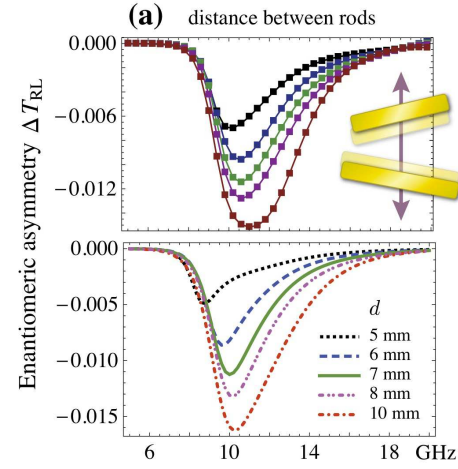

(b) rod size difference

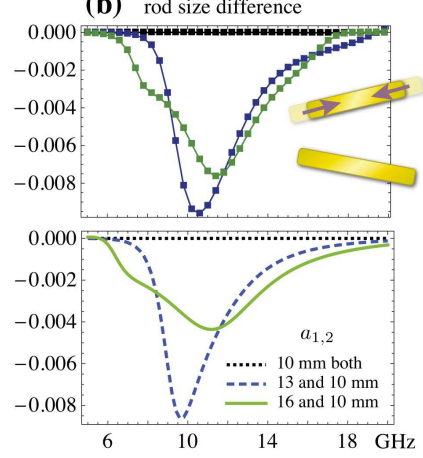

(c) rod misalignment

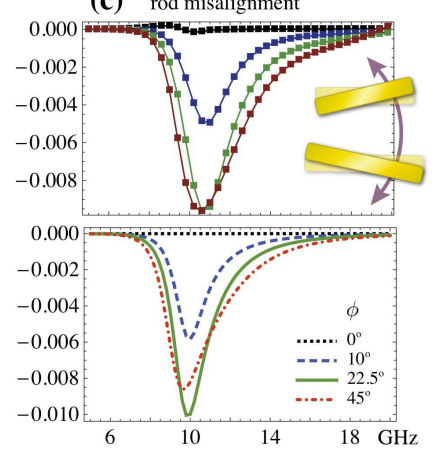

(d) rod displacement

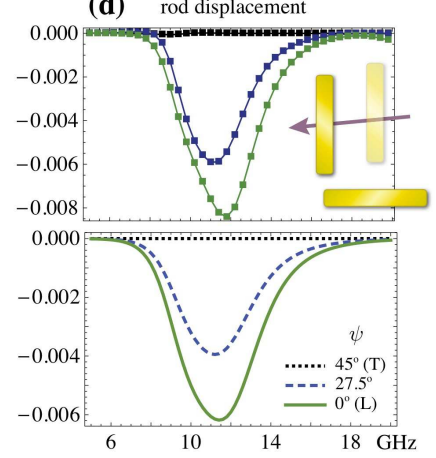

Figure 4. (Color online) Numerical (top) and analytical (bottom) dependence of $\Delta T_{R L}$ on (a) inter-rod distance $d$, (b) difference in rod length $a_{1}-a_{2}$, (c) rod misalignment angle $\phi$, and (c) rod displacement angle $\psi$ for $\phi=90^{\circ}$ (as the dimer changes between T- and L-shaped). Unless specified otherwise, $a_{1}=13 \mathrm{~mm}, a_{2}=10 \mathrm{~mm}, d=6 \mathrm{~mm}, \phi=45^{\circ}$, and $\psi=0^{\circ}$.

$\left|C_{1}\right|=\left|C_{2}\right|$. However, in the spectral range of strong absorption the coupling of RH and $\mathrm{LH}$ incident waves to the crystal eigenmodes $\mathbf{h}_{1,2}$ is very different $\left(C_{1,2}^{R} \neq C_{1,2}^{L}\right)$, leading to planar chirality in the form of enantiomeric asymmetry in the transmisison $\Delta T_{R L}=T_{R}-T_{L}$ (Fig. 3 $\mathrm{d}$ ).

Such a difference can be used to quantify the "strength" of planar chiral properties in a particular structure. Fig. 4 shows the spectra $\Delta T_{R L}$ for various shapes of the dimer, analytical results from Eqs. (1)-(4) compared to the results of direct 3D frequency-domain numerical simulations ${ }^{11}$. It can be seen that the effective medium model offers a good coincidence with numerical results.

Most rod dimers are seen to exhibit chiral properties unless the dimer has an in-plane mirror symmetry, which makes the 2D enantiomers indistinguishable and therefore enforces $\Delta T_{R L}=0$. For the geometrical tansformation considered, such symmetry is achieved in the following cases: (i) for rods of equal length (V-shaped dimer, Fig. 4b); (ii) for parallel rods (II-shaped dimer, Fig. 4k); (iii) for a T-shaped dimer (Fig. 4d). In all these cases, the analytical model correctly predicts the absence of enantiomeric asymmetry. Otherwise, chiral properties appear to be stronger when enantiomers are most distinct, so there is an optimum rod misalignment angle $\phi \simeq 22.5^{\circ}$ in Fig. 4k. It is also necessary that the resonances of the individual rods have some degree of spectral overlap. Hence, $\Delta T_{R L}$ depends on the length mismatch between the rods in a non-monotonic way: it first increases when the dimer deviates from the achiral $\mathrm{V}$ shape, reaches the maximum value, and then decreases again with a pronounced resonance splitting as $\Delta a$ becomes greater (see Fig. 4b). Finally, $\Delta T_{R L}$ becomes smaller as the distance between the rods de-

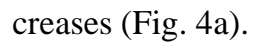

Since the coupling between meta-atoms in the 2D lattice was neglected both in the analytical model and in the simulations, it can be concluded that planar chirality in the PCMs under study is intrinsic (i.e., attributable to the geometry of the meta-atom itself) rather than extrinsic (attributable to the meta-atom arrangement, e.g., in tilted-cross arrays ${ }^{12}$ ). This agrees with earlier time-domain simulation results ${ }^{13}$. With the proposed model extended to include the inter-atom coupling, explicit account of intrinsic vs. extrinsic effects in PCMs can be given. Combining the intrinsic and extrinsic contribution in the same PCM can be used to maximize its chiral properties.

In conclusion, we have shown that plasmonic rod dimers act as elementary planar chiral meta-atoms. Using a simple coupled-dipole analytical approach, we show the presence of elliptical dichroism and enantiomeric asymmery. We have explored the chiral properties of rod dimers of different geometries and demonstrated that symmetric dimers are achiral, whereas the structures with most distinct $2 \mathrm{D}$ enantiomers have the most pronounced chiral properties. Analytical results are in good agreement with direct frequency-domain numerical simulations.

This work was supported in part by the Deutsche Forschungsgemeinschaft (FOR 557) and the Natural Sciences and Engineering Research Council of Canada (NSERC).

\section{REFERENCES}

${ }^{1}$ M. Kuwata-Gonokami, N. Saito, Y. Ino, M. Kauranen, K. Jefimovs, T. Vallius, J. Turunen, and Y. Svirko, Phys. Rev. Lett. 95, 227401 (2005).

${ }^{2}$ V. A. Fedotov, P. L. Mladyonov, S. L. Prosvirnin, A. V. Rogacheva, Y. Chen, and N. I. Zheludev, Phys. Rev. Lett. 97,167401 (2006).

${ }^{3}$ E. Plum, V. A. Fedotov, and N. I. Zheludev, Appl. Phys. Lett. 94, 131901 (2009).

${ }^{4}$ J. Petschulat, C. Menzel, A. Chipouline, C. Rockstuhl, A. Tünnermann, F. Lederer, and T. Pertsch, Phys. Rev. A 78, 043811 (2008).

${ }^{5}$ Z.-G. Dong, H. Liu, M.-X. Xu, T. Li, S.-M. Wang, S.-N. Zhu, and X. Zhang, Opt. Express 18, 18229 (2010).

${ }^{6}$ S. V. Zhukovsky, V. M. Galynsky, and A. V. Novitsky, Opt. Lett. 34, 19881990 (2009).

${ }^{7}$ A. Moroz, J. Opt. Soc. Am. B 26, 517-527 (2009).

${ }^{8}$ F. I. Fedorov, Theory of Gyrotropy (Minsk, Nauka i Tekhnika, 1976).

${ }^{9}$ L. M. Barkovsky, Sov. Phys. Crystallogr. 21, 245-247 (1976).

${ }^{10}$ G. N. Borzdov, J. Math. Phys. 38, 6328-6366 (1997).

${ }^{11}$ Ansys HFSS, http://www.ansoft.com/products/hf/hfss/

${ }^{12}$ S. N. Volkov, K. Dolgaleva, R. W. Boyd, K. Jefimovs, J. Turunen, Y. Svirko, B. K. Canfield, and M. Kauranen, Phys. Rev. A 79, 043819 (2009).

${ }^{13}$ C. Kremers, S. V. Zhukovsky, and D. N. Chigrin, in Theoretical and Computational Nanophotonics: Proc, of the 2nd International Workshop, D. N. Chigrin, ed., Vol. 1176 of AIP Conf. Proc. Series (AIP, Melville, NY, 2009), pp. 118-120. 